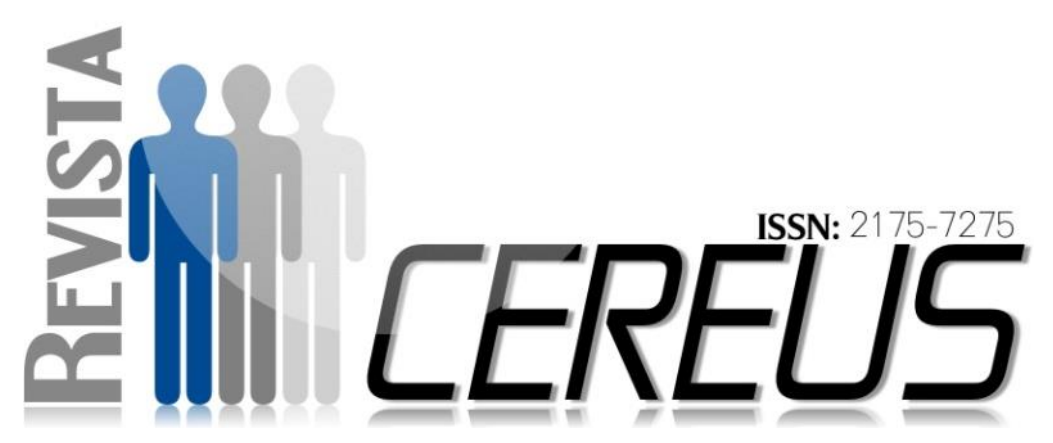

\title{
PERFIL MOTOR DE IDOSOS: EXISTE EXERCÍcIO FísICO MAIS
} EFICAZ?

VENÂNCIO, Patrícia Espíndola Mota ${ }^{1}$ SILVA, Sidnei Sanches Nascimento ${ }^{1}$ OLIVEIRA-SILVA, Iransé ${ }^{1}$

\section{RESUMO}

Objetivo: Verificar a relação entre o perfil motor de idosos sedentários e ativos, participantes de diferentes programas de exercício físico. Métodos: A amostra foi composta por 45 idosos divididos em três grupos (praticantes de hidroginástica - GH; praticantes de musculação - GM; idosos que não faziam um programa regular de exercício físico - GS), os quais realizaram testes motores que avaliavam a coordenação motora, equilíbrio, esquema corporal, estruturação espacial e temporal. Para a realização dos testes, foi utilizada a bateria motora de Rosa Neto (2007). Resultados: $\mathrm{Na}$ comparação entre grupos não houve diferenças significativas, porém, observa-se que os idosos praticantes de musculação estão aparentemente melhores que os demais participantes. $E$ quando comparadas as 
diferenças entre os sexos os homens estão significativamente melhores que as mulheres nas classificações de esquema corporal, estruturação espacial, estruturação temporal e na classificação geral. Conclusão: Entre os grupos sedentário e ativo (musculação e hidroginástica) houve diferença significativa. Contudo ficou evidenciado que os idosos praticantes de musculação mostraram-se melhores que os demais participantes. E quando comparados entre os sexos, os homens estão significativamente melhores que as mulheres.

Palavras-chave: Idosos. Psicomotricidade. Exercícios Físicos.

\section{MOTOR PROFILE OF ELDERLY: IS THERE MORE EFFICIENT PHYSICAL EXERCISE?}

\section{ABSTRACT}

Aim: To verify the relationship between the motor profile of sedentary and active elderly, participants of different physical exercise programs. Methods: The sample consisted of 45 elderly people divided into three groups (water aerobics - WG, weight training - WT, elderly people who did not participate in a regular physical exercise program - SG), who underwent motor tests that evaluated motor coordination, balance, Body schema, spatial and temporal structuring. To perform the tests, the motor battery of Rosa Neto (2007) was used. Results: In the comparison between groups there were no differences, with only differences in percentiles, where weight training seniors are apparently better than the other participants. But when comparing the differences between men sexes are significantly better than women in body image classifications, spatial structure, temporal structure and overall. Conclusion: Among the sedentary and active groups (weight training and water aerobics) there was a significant 
difference. However, it was evidenced that the elderly practicing weight training proved to be better than the other participants. And when compared between the sexes, men are significantly better than women.

Key Words: Elderly. Psychomotor. Physical Exercises. 


\section{INTRODUÇÃO}

A psicomotricidade é a educação dos movimentos por meio de relações com os processos cerebrais, a qual interage a afetividade e o pensamento, buscando melhorar 0 potencial cognitivo (CAMARGOS; MACIEL, 2016). Seu principal objeto de estudo é o corpo em movimento, com intuito de inserir e/ou manter inserido o indivíduo na sociedade, da infância à maturidade, por meio do desenvolvimento motor, pessoal e cognitivo (LE BOULCH, 2007; RABELO; AQUINO, 2014).

É cabível salientar que desde as fases mais tenras até a maturidade, é possível à aquisição e aperfeiçoamento de valências físicas, bem como os processos mentais (e.g. coordenação motora, tônus muscular, desenvolvimento intelectual e noções espaço-temporais)

e

esse aperfeiçoamento depende diretamente de estímulos psicomotores que 0 indivíduo recebe (WALLY et al., 2010; ROSSI, 2012; OLIVEIRA-SILVA e ARCANJO, 2017).

Por este motivo é de suma importância a aplicação das práticas motoras na população idosa, uma vez que, nessa fase da vida o corpo passa por diversas mudanças em diferentes contextos, que intervêm na realização de tarefas diárias básicas, e tende a comprometer a locomoção e consequentemente a independência funcional (GONÇALVES et al., 2013).

É importante salientar que o declínio funcional é algo natural e esperado (RIBEIRO et al., 2015), entretanto pessoas idosas são propensas a sofrer mais em função deste declínio, especialmente devido a falta de estímulos, diminuição do contato familiar e déficit cognitivo decorrente do avançar da idade (MIRANDA et al., 2015; OLIVEIRA; MATTOS, 2012).

Estudo prospectivo (DALY et al, 2013) demonstrou que intervenções relacionadas ao equilíbrio e a velocidade da marcha, que podem advir por meio de um programa de exercício físico ou mesmo atividade física, devem ser iniciadas o quanto antes, especialmente porque a degeneração do sistema musculoesquelético acontece gradativamente pelo processo fisiológico do envelhecimento, envolvendo fatores neuromotores (redução da velocidade da condução nervosa, diminuição da ativação 
muscular, mudanças na postura e no movimento) e musculoesqueléticos, como sarcopenia e osteopenia. Estes fatores relacionam-se diretamente com a diminuição da atividade física na vida do idoso (BAKER et al., 2010; FOONG et al., 2016). Vale destacar que não está bem estabelecido na literatura qual programa de exercício ou atividade física é mais eficaz para o perfil motor de idosos.

As perdas funcionais claramente afetam a qualidade de vida dos idosos. Considerando isso, Santos et al. (2006) já atentaram que eram poucas as políticas públicas que objetivam promover o bem-estar biopsicossocial

\section{METODOLOGIA}

O estudo teve desenho descritivo correlacional, e contou com a participação de 45 idosos com idade compreendida entre 64 e 72 anos residentes na cidade de Goianésia-GO. A amostra foi de conveniência e selecionada para compor 3 grupos, cada um com 15 participantes, sendo 1 grupo sedentário (GS) e 2 grupos ativos [praticantes de musculação (GM) e praticantes de hidroginástica $(\mathrm{GH})]$. Para participar do estudo o idoso deveria frequentar 0 Centro de de idosos, e uma década depois este cenário ainda persiste (FOCHAT et al., 2016). Neste sentido é importante entender como minimizar estas perdas, pois estamos diante de um cenário crescente desta população no Brasil (VAZ, 2013) e no mundo (UNITED NATIONS, 2015), e os custos para o estado garantir a sobrevida destas pessoas é elevado (FOCHAT et al., 2016).

Desse modo, esse trabalho teve por objetivo verificar a relação entre o perfil motor de idosos sedentários e ativos, participantes de diferentes programas de exercício físico.

Convivência de Idosos (CCl) da cidade a pelo menos seis meses; não apresentar nenhuma patologia limitante do movimento; aceitar realizar os testes motores propostos. Seriam excluídos aqueles que não realizassem todos os testes.

Os participantes foram classificados em grupos de atividade de acordo com as atividades que faziam: Grupo Sedentário (GS), não participavam das atividades físicas sistematizadas; Grupos fisicamente 
ativos (GM; $\mathrm{GH})$ praticavam a respectiva atividade há um tempo mínimo de 6 meses em 3 sessões semanais sistematizadas.

Este estudo foi aprovado pelo Comitê de Ética do Centro Universitário de Anápolis - UniEVANGÉLICA (protocolo: 1.105.284)

Após a seleção dos idosos, foi realizado um agendamento individual para a realização dos testes motores, em um espaço público utilizado para a convivência dos idosos, na cidade de Goianésia. A duração das avaliações foi $\sim 45$ minutos.

A Bateria psicomotora de Rosa Neto (2007), uma ferramenta validada e reconhecidamente capaz de avaliar a motricidade, é composta por testes de Coordenação Motora Global (CMG), Equilíbrio (EQ), Esquema Corporal (EC), Estruturação Espacial (EE) e Estruturação Temporal (ET) e Classificação Geral da bateria (CG). O

\section{RESULTADOS}

A tabela 1 refere-se aos resultados obtidos pelos testes aplicados para os componentes, Coordenação motora (CM), Equilíbrio (EQ), Esquema Corporal (EC), Estruturação Espacial (EE), teste é realizado em onze níveis e para cada nível existe uma pontuação e classificação: Muito inferior (MI) $\leq 69$ pontos; inferior (IF) $\geq 70 \leq 79$ pontos; Normal baixo (NB) $\geq 80 \leq 89$ pontos; Normal médio (NM) $\geq 90 \leq 109$ pontos; Normal alto (NA) $\geq 110 \leq 119$ pontos; Superior (SP) $\geq 120 \leq 129$ pontos; muito superior $(\mathrm{MS}) \geq 130$ pontos.

\section{Análise Estatística}

Para verificar a normalidade da distribuição dos dados foi utilizado o teste de Kolmogorov-Smirnov. Os dados são apresentados através da estatística descritiva, utilizando-se do percentual. O teste de Kruskal Wallis foi utilizado para verificar as diferenças entre as atividades (GC; GM; GH). O teste de Mann-Whitney foi utilizado para verificar as diferenças entre sexos.

Utilizou-se o software SPSS for Windows versão 21.0, e o nível de significância adotado foi de $\mathrm{P} \leq 0,05$.

Estruturação Temporal (ET) e a Classificação Geral (CG) quanto ao aspecto psicomotor. Constatando uma diferença significativa entre os grupos avaliados na CM, EQ, EC e CG, podendo ser observado que em três 
desses quatro componentes $(\mathrm{CM}, \mathrm{EQ}$, CG) os praticantes de musculação foram melhores que os demais. Destaca-se que no componente EC, os grupos ativos (GH, GM) obtiveram melhor classificação. Contudo é importante observar que a $\mathrm{CM}$ e $\mathrm{EQ}$ daqueles que faziam hidroginástica apresentaram os piores resultados.

Tabela 1. Classificação \% dos idosos quanto aos componentes motores

\begin{tabular}{|c|c|c|c|c|c|c|c|c|}
\hline \multirow{2}{*}{\multicolumn{2}{|c|}{ Componente }} & \multicolumn{7}{|c|}{ Classificação \% } \\
\hline & & $\mathrm{Ml}$ & IF & NB & NM & NA & SP & MS \\
\hline \multirow{3}{*}{ CM } & $\mathrm{GH}$ & 20 & 20 & 26,7 & 26,7 & 6,7 & - & - \\
\hline & GM & - & 13,3 & 6,7 & 13,3 & 20 & 26,7 & $20^{*}$ \\
\hline & GS & 6,7 & 13,3 & 13,3 & 33,3 & 20 & 13,3 & 6,7 \\
\hline \multirow{3}{*}{ EQ } & $\mathrm{GH}$ & 20 & 20 & 26,7 & 26,7 & 6,7 & - & - \\
\hline & GM & & 13,3 & 6,7 & 13,3 & 20 & 26,7 & $20^{*}$ \\
\hline & GS & 6,7 & 13,3 & 13,3 & 33,3 & 20 & 13,3 & 6,7 \\
\hline \multirow{4}{*}{ EC } & $\mathrm{GH}$ & - & - & - & - & 13,3 & 46,7 & $40^{*}$ \\
\hline & GM & - & - & - & - & 20 & 60 & $20^{*}$ \\
\hline & GS & - & - & - & - & 40 & 60 & - \\
\hline & $\mathrm{GH}$ & 13,3 & 6,7 & - & 20 & - & 26,7 & 33,3 \\
\hline \multirow{2}{*}{ EE } & GM & & 13,3 & - & 20 & 20 & 46,7 & - \\
\hline & GS & 6,7 & 26,7 & - & 20 & - & 20 & 26,7 \\
\hline \multirow{3}{*}{ ET } & $\mathrm{GH}$ & - & 13,3 & - & 6,7 & 26,7 & 40 & 13,3 \\
\hline & GM & - & 6,7 & - & - & 26,7 & 46,7 & 20 \\
\hline & GS & - & - & - & 20 & 13,3 & 33,3 & 33,3 \\
\hline \multirow{3}{*}{ CG } & $\mathrm{GH}$ & & 6,7 & 60 & 33,3 & - & - & - \\
\hline & GM & - & - & 6,7 & 73,3 & $20^{*}$ & - & - \\
\hline & GS & & 6.7 & 46,7 & 46.7 & - & - & - \\
\hline
\end{tabular}

Muito Inferior (MI), Inferior (IF), Normal Baixo (NB), Normal Médio (NM), Normal Alto (NA), Superior $(\mathrm{SP})$, Muito Superior (MS); Coordenação Motora (CM), Equilíbrio (EQ), Esquema Corporal (EC), Estruturação Espacial (EE), Estruturação Temporal (ET) e a Classificação Geral (CG), para os grupos: praticantes de Hidroginástica (GH), praticantes de Musculação (GM) e de idosos Sedentários (GS). Significância $\leq 0,05\left(^{*}\right)$.

Quanto à classificação \% da diferença entre sexos, levando em consideração os componentes, EC, EE, ETe CG, os dados são apresentados na tabela 2. Foi mostrado que os homens apresentaram melhor classificação \% de forma significativa nos componentes analisados de forma individual e consequentemente no aspecto psicomotor geral. 
Tabela 2. Classificação \% dos componentes psicomotores de idosos.

\begin{tabular}{|c|c|c|c|c|c|c|c|c|}
\hline \multirow{2}{*}{\multicolumn{2}{|c|}{ Componente }} & \multicolumn{7}{|c|}{ Classificação \% } \\
\hline & & $\mathrm{MI}$ & IF & NB & NM & NA & SP & MS \\
\hline \multirow{2}{*}{ EC } & M & - & - & - & 3,7 & 11,1 & 55,6 & $29,6^{*}$ \\
\hline & $\mathrm{F}$ & 3 & - & - & 3 & 30,3 & 48,5 & 15,2 \\
\hline \multirow{2}{*}{ EE } & $\mathrm{M}$ & 3,7 & 7,4 & - & 11,1 & - & 25,9 & $51,9^{*}$ \\
\hline & $\mathrm{F}$ & 6,1 & 18,2 & - & 21,2 & - & 27,3 & 27,3 \\
\hline \multirow{2}{*}{ ET } & $\mathrm{M}$ & - & - & - & 3,7 & 25,9 & 40,7 & $29,6^{*}$ \\
\hline & $\mathrm{F}$ & 6,1 & 9,1 & 9,1 & 15,2 & 21,2 & 30,3 & 9,1 \\
\hline \multirow{2}{*}{ CG } & $\mathrm{M}$ & - & 3,7 & 29,6 & $59,3^{*}$ & 7,4 & - & - \\
\hline & $\mathrm{F}$ & - & 12,1 & 48,5 & 36,4 & 3 & - & - \\
\hline
\end{tabular}

Muito Inferior (MI), Inferior (IF), Normal Baixo (NB), Normal Médio (NM), Normal Alto (NA), Superior (SP), Muito Superior (MS); Esquema Corporal (EC), Estruturação Espacial (EE), Estruturação Temporal (ET) e a Classificação Geral (CG); Masculino (M), Feminino (F).

\section{DISCUSSÃO}

Os resultados demonstram 0 efeito da prática de exercício físico sistematizado no desenvolvimento motor de idosos. A participação em um programa de exercício físico sistematizado que envolve 0 desenvolvimento de força pode influenciar no perfil psicomotor desta população. Para a amostra estudada, os homens apresentam uma melhor capacidade motora que as mulheres.

A literatura evidencia o papel dos programas regulares de exercício físico na coordenação motora de idosos (KATZER et al., 2012; NASCIMENTO et al., 2013; FATORI et al., 2015), contudo o presente estudo além de reforçar esta afirmativa, demonstra que um programa de exercício físico regular com ênfase na força, a exemplo da Musculação, pode contribuir de maneira mais efetiva com a coordenação motora, equilíbrio e consequentemente na classificação geral da motricidade de idosos, conforme apresentado por OliveiraSilva et al. (2017). Isso se dá porque a força é um componente importante na manutenção de níveis satisfatórios de independência funcional, impactando diretamente na qualidade de vida dos idosos (FURTADO et al., 2016).

Destaca-se que mesmo não tendo sido mensurado o nível de força da amostra estuda, estudo prévio (RUBIRA et al., 2014), evidencia que 
maiores níveis de força melhoram a velocidade de oscilação, a oscilação anteroposterior e a velocidade da marcha, componentes que auxiliam no dinamismo e no equilíbrio da marcha, impactando no equilíbrio, e prevenindo o risco de queda (DA SILVA et al., 2014). Vale destacar que idosos que apresentam limitações dos movimentos, passam a ser dependentes para executar as atividades da vida diária (AVDs), e consequentemente passa a depender de um cuidador (FABRÍCIO-WEHBE, et al. 2016), onerando consideravelmente a família, que muitas vezes opta por a procurar as instituições de longa permanência que cuidam de idosos (DEL DUCA et al., 2012; HOLANDA, 2012; FABRÍCIO-WEHBE et al., 2016; COLLAMATI et al., 2016). Daí a importância de conscientizar o idoso da necessidade de manter sua capacidade funcional.

Os resultados do presente estudo referentes ao esquema corporal mostram que esta talvez tenha sido a valência com melhor classificação, independente do grupo avaliado, mesmo os sedentários, e que os homens apresentam-se melhores nessa valência do que as mulheres de acordo com os parâmetros de classificação adotados. Em um estudo que analisou o comportamento do esquema corporal da adolescência à terceira idade, foi demonstrado que com o passar dos anos no que tange a representação do esquema corporal há um decréscimo de percepção quanto às proporções reais (PEREIRA et al., 2012). Fonseca et al. (2012) analisaram os benefícios de exercícios de estimulação perceptual sobre 0 esquema corporal de idosos, exercícios que abrangem características psicomotoras. Foram achados do estudo que esses exercícios podem levar os idosos a melhor compressão do próprio corpo e influenciar na auto aceitação, podendo assim esses exercícios ser uma alternativa para idosos que apresentam déficits no que tange o esquema corporal.

Em um estudo que comparou o tempo de reação entre adolescentes com idade escolar e idosos através de simples teste clínico, foi mostrado que a idade retarda a reação motora do idoso (GONÇALVES et al., 2013). No presente estudo, as estruturações espaciais e temporais apresentam relação direta com o tempo de reação. Não foram evidenciadas diferenças entre os grupos pesquisados, contudo ao comparar os sexos, os dados 
sugerem que os homens idosos apresentam-se melhor qualificados nessas capacidades.

Este estudo limitou a verificar a relação entre o perfil motor de idosos

\section{CONSIDERAÇÕES FINAIS}

A partir da análise dos dados separados por diferentes grupos, foi possível concluir que, quando comparados os idosos entre os grupos (ativo e sedentário) houve uma diferença significativa, que confirma que os ativos apresentam melhor perfil motor que os sedentários na maioria dos componentes testados. Contudo ficou evidenciado que os idosos praticantes de musculação mostraramse melhores que os demais participantes na maioria dos componentes. Em relação aos sexos, os homens estão significativamente melhores que as mulheres nas sedentários e participantes dos programas de hidroginástica ou musculação da cidade de Goianésia e frequentadores do $\mathrm{CCl}$ local.

classificações de esquema corporal, estruturação espacial, estruturação temporal e na classificação geral.

Todavia é sugestível a elaboração de mais estudos para este mesmo tema, afim de maior aprofundamento teórico-cientifico sobre a psicomotricidade de idosos. Porém é importante destacar a esta população (i.e. idosos) e os profissionais que prescrevem exercícios físicos para eles, que os exercícios de força, com destaque para a musculação, deve ser uma alternativa considerada.

\section{REFERÊNCIAS}

BAKER, Laura D.; FRANK, Laura L.; FOSTER-SCHUBERT, Karen, et al. Effects of Aerobic Exercise on Mild Cognitive Impairment: A Controlled Trial. Archives of neurology. v. 67, n.1, Jan. 2010, p. 71-79.

CAMARGOS, Ellen Kassia de; MACIEL, Rosana Mendes. The importance of psychomotricity in children education. Multidisciplinary Core scientific journal of knowledge. v.9. Oct/Nov 2016, p. 254-275. 
COLLAMATI, Agnese; MARTONE, Anna Maria; POSCIA, Andrea, et al. Anticholinergic drugs and negative outcomes in the older population from biological plausibility to clinical evidence. Aging Clin Exp Res, v. 28, n. 1, 2016, p. 25-35.

DA SILVA, Juliana Rosini; TRINDADE CAMARGO, Regina Celi; NUNES, Maurillo Maroco, et al. Análise da alteração do equilíbrio, da marcha e o risco de queda em idosos participantes de um programa de fisioterapia. Rev e-ciência. v. 2, n. 2, 2014, p. 11-18.

DALY, Robin M; ROSENGREN, Bjorn E; ALWIS, Gayani, et al. Gender specific agerelated changes in bone density, muscle strength and functional performance in the elderly: a-10 year prospective population-based study. BMC Geriatr. v.13, n.1, 2013, p. 1-9.

DEL DUCA, Giovâni Firpo; SILVA, Shana Ginar; THUMÉ, Elaine, et al. Indicadores da institucionalização de idosos: estudo de casos e controles. Rev Saúde Pública. v. 46, n.1, 2012, p. 147-153.

FABRÍCIO-WEHBE, Suzele Cristina Coelho; RODRIGUES, Rosalina Aparecida Partezani; HAAS, Vanderlei Jose, et al. Association of frailty in hospitalized and institutionalized elderly in the community dwelling. Rev Bras Enferm, v. 69, n. 4, 2016, p. 644-649.

FATORI, Camila de Oliveira; LEITE, Camila Ferreira; SOUZA, Luciane Aparecida Pascussi Sande de, et al. Dupla tarefa e mobilidade funcional de idosos ativos. Rev. Bras. Geriatr. Gerontol. Rio de Janeiro, v.18, n.1, 2015, p. 29-37.

FOCHAT, Romário Costa; POLONINI, Hudson Caetano; BARBOSA, Elaine Maria Silva, et al. Prevalências de doenças de grande impacto na saúde pública em idosos frágeis residentes na zona da mata Mineira-Brasil: um estudo de base populacional. Rev. APS. v.19, n.2, abr/jun 2016, p. $268-276$.

FONSECA, Cristiane Costa; GAMA, Eliane Florêncio; THURM, Bianca Elisabeth, et al. Benefícios da estimulação perceptual corporal no esquema corporal de idosos. Rev Bras Geriatr Gerontol. v.15, n.2, 2012, p.353-364.

FOONG, Yi Chao, CHHERAWALA, Nabil, AITKEN, Dawn, et al. Acelerometer determined physical activity muscle mass, and leg strength in community- dwelling older adults. Jornal of Cachexia, Sarcopenia and Muscle. v. 7, 2016, p. 25-283.

FURTADO, Guilherme Eustáquio; UBA CHUPEL, Matheus; CARVALHO, Humberto $M$, et al. A Effect of a chair - yoga exercises on stress hormone levels, daily life activities falls end physical fitness in institutionalized older adults. Complementary therapies in Clinical Practice. v. 24, 2016, p. 123-129. 
GONÇALVES, Daianne; MENEZES, Jefferson; MACHADO, Alvaro Sosa, et al. Comparação do tempo de reação simples de idosos e adolescentes. BIOMOTRIZRevista Científica da Univ Cruz Alta. v.7, n.1, Jul/2013, p. 52-62.

HOLANDA, Cristina Marques de Almeida; GUERRA, Ricardo Oliveira; NÓBREGA, Patrícia Vidal de Negreiros, et al. Salivary cortisol and frailty syndrome in elderly residents of long-stay institutions a cross-sectional study. Arch Gerontol Geriatr. v. 54 , n. 2, 2012, p. 146-151.

KATZER, Juliana Izabel; LEDUR ANTES, Danielle, CORAZZA, Sara Teresinha. Coordenação motora de idosas. ConScientiae saúde. v.11, n. 1, 2012, p.159-163

LE BOULCH, Jean. Educação psicomotora: a psicocinética na idade escolar. Artmed, 2007.

MIRANDA, Amanda Cristina de Carvalho; SÉRGIO, Samanta Rodrigues; FONSECA, Grazielle Neves Soares, et al. Avaliação da presença de cuidador familiar de idosos com déficits cognitivo e funcional residentes em Belo Horizonte - MG. Rev. Bras. Geriatr. Gerontol. Rio de Janeiro, v.18, n.1, 2015, p.141-150.

NASCIMENTO, Carla Manuela Crispim; AYAN, Carlos; CANCELA, José Maria, et al. Exercícios físicos generalizados capacidade funcional e sintomas depressivos em idosos brasileiros. Rev Bras Cineantropometria Desempenho Hum. v. 15, n. 4, 2013, p.486-497.

OLIVEIRA, Paulo Henrique de; MATTOS, Inês Echenrique. Prevalência e fatores associados à incapacidade funcional em idosos institucionalizados no município de Cuiabá, Estado de Mato Grosso, Brasil, 2009-2010. Epidemiologia e Serviços de Saúde. Brasília, v.21, n. 3, Set. 2012, p.395-406.

OLIVEIRA-SILVA Iransé, ARCANJO, Larissa Raphaella Bardella. Papel da hidroginástica na capacidade funcional de idosos. Rev. Educ. Saúde. v.5, n 1, 2017, p. 15-20.

OLIVEIRA-SILVA, Iransé; GONÇALVES, Helton Ramos; VENÂNCIO, Patrícia Espíndola Mota, et al. Influence of resistance training in quality of life, body composition, and physical performance of community-dwelling elderly women. MTP \& Rehab Journal. v.15, 2017, p, 510-517.

PEREIRA, Emy Suelen; SEGHETO, Wellington; MIRANDA, Maria Luiza de Jesus, et al. Comportamento do esquema corporal do adolescente ao idoso. Rev Bras Ciências do Envelhec Hum. v.7, supl. 1, 2012, p. 20-28.

RABELO, Kalícia Ingrid de Lacerda; AQUINO, Gisele Braga de. Relação entre psicomotricidade e desenvolvimento infantil: um relato de experiência. Revista Científica Faminas. v. 10, n. 3, Set-Dez, 2014, p.109-123. 
RIBEIRO, Dâmarys Kohlbeck de Melo Neu; LENARDI, Maria Helena; MICHEL, Tatiane, et al. Fatores contributivos para a independência funcional de idosos longevos. Revista da Escola de Enfermagem da USP. v.49, n.1, 2015, p.89-95.

ROSA NETO, Francisco. Manual de avaliação motora para terceira idade. Artmed; 2007.

ROSSI, Francieli Santos. Considerações sobre a Psicomotricidade na Educação Infantil. Ministério da Educação Universidade Federal dos Vales do Jequitinhonha e Mucuri - UFVJM Minas Gerais - Brasil. Revista Vozes dos Vales. n.1, ano 1, Mai/ 2012, p. 1-18.

RUBIRA, Ana Paula Fernandes de Angelis; GONÇALVES DA SILVA, Maria; GOIS DE CARVALHO, Thiago, et al. Efeito de exercícios psicomotores no equilíbrio de idosos. ConScientiae Saúde. v. 13, n. 1, 2014, p.54-61.

SANTOS, Luciane de Medeiros; DA ROS, Marco Aurélio; CREPALDI, Maria Aparecida, et al. Grupos de promoção à saúde no desenvolvimento da autonomia, condições de vida e saúde. Rev. Saúde Pública. v. 40, n. 2, 2006, p.346-352.

UNITED NATIONS, Department of Economic and Social Affairs, Population Division. World Population Ageing. United Nations, New York, 2015, p. 1-164.

VAZ, Fabiane de Castro; MOLINA, Guilherme Eckhardt; PORTO, Luiz Guilherme Grossi, et al. Cortisol e Atividade Física: será o estresse um indicador do nível de atividade física espontânea e capacidade física em idosos? Brasília Med. v. 50, n. 2, 2013, p. 143-152.

WALLY, Raquel; KAEFER, Angélica; ÁVILA, Luciana Toaldo Gentilini, et al. Comportamento motor de idosos: subsídios para a intervenção profissional nesta população. In: Mariângela da Rosa Afonso; Adriana Schüler Cavalli. (Org.). Trabalhando com a terceira idade: trajetórias de intervenção. Pelotas: Editora e Gráfica UFPEL, 2010, p. 154-171. 